\title{
INVESTIGACIÓN/RESEARCH
}

\section{EMPODERAMIENTO DE ENFERMERÍA COMO PROFESIÓN Y SUJETO SOCIAL: CASO CHIHUAHUA}

Leticia Moriel-Corral' : Universidad Autónoma de Chihuahua. México Imoriel@uach.mx

Norma Pizarro: Universidad Autónoma de Chihuahua. México npizarro@uach.mx

Hilda Hernández-Hernández: Universidad Autónoma de Chihuahua. México hhernand@uach.mx

Elizabeth Bonilla-Loyo: Universidad Veracruzana. México lizitabon@yahoo.com.mx

\section{RESUMEN}

El empoderamiento de las enfermeras en el desarrollo disciplinar es un reto que persiste desde su origen. El objetivo de esta investigación fue identificar la percepción y el nivel de empoderamiento de las enfermeras que trabajan en los hospitales públicos y privados en el estado de Chihuahua. Metodología: Se realizó un estudio cualitativo a través del paquete tecnológico-metodológico Grupos de Discusión [GD], el cual permitió reflexionar sobre el sentido de la enfermería como sujeto social y como profesión. Fue un estudio descriptivo y transversal, participaron cuatro grupos de discusión, dos grupos de enfermeras(os) en activo, y dos de estudiantes de primeros y últimos semestres licenciatura. Se diseñó un protocolo metodológico con su respectivo instrumento centrado en tres campos semánticos con sus detonadores. Resultados: Se identificó evolución de la enfermería, como profesión logra altos grados académicos, donde demuestra dominio, competencia y obtiene reconocimiento profesional por decreto gubernamental. Estos resultados se analizaron en tres categorías: Oficio, Subordinación y Reconocimiento social, las enfermeras se posicionaron, lograron reconocimiento en hospitales públicos y en el ámbito

\footnotetext{
${ }^{1}$ Autor correspondiente Leticia Moriel Corral.

Docente de la Maestría en Enfermería de la Facultad de Enfermería y Nutriología de la Universidad Autónoma de Chihuahua de México. Integrante del Cuerpo Académico 102.
}

Correo: Imoriel@uach.mx 
comunitario, ejerciendo el rol cuidador, educador y administrador principalmente, se perciben así mismas empoderadas, por su motivación y su saber sobre el cuidado a pesar de los obstáculos políticos. Análisis y Discusión: La enfermera debe actuar con compromiso individual, como una necesidad de empoderamiento de la profesión en beneficio de la sociedad. Conclusiones. Las enfermeras no explicitan experiencias de empoderamiento, sin embargo a través de sus diálogos y discursos relatan e identifican vivencias que corresponden a atributos del empoderamiento.

PALABRAS CLAVE: Empoderamiento - Cuidado - Funciones - Enfermera y Enfermería

\section{NURSING EMPOWERING AS PROFESSION AND SOCIAL OBJECT: CASE CHIHUAHUA}

\section{ABSTRACT}

The empowerment of nurses in the development discipline is a challenge that persisted from the beginning. The objective of this study was to identify the level of awareness and empowerment of nurses working in public and private hospitals in the state of Chihuahua. Methodology: A qualitative study was conducted through technological and methodological package Discussion Groups [GD], which allowed to reflect on the meaning of nursing as a social subject and as a profession. It was a descriptive study, involving four focus groups, two groups of nurses active, and two students of first and last semesters degree. Methodological protocol was designed with its instrument focused on three semantic fields with their detonators. Results: We identified evolution of nursing as a profession achieves high degrees, which demonstrates mastery, competence and professional recognition obtained by government decree. These results were analyzed in three categories: Office, Subordination and social recognition, nurses were positioned, achieved recognition in public hospitals and community level, exercising the role of caregiver, educator and administrator mainly perceived empowered themselves, for their motivation and know about caring despite the political obstacles. Discussion: The nurse must act with individual commitment, as a need to empower the profession to benefit society. Conclusions. Nurses do not explicitly empowering experience, however through their dialogues and discourses relate and identify experiences that correspond to attributes of empowerment.

KEY WORDS: Empowerment - Care - Roles - Nurse and Nursing

\section{INTRODUCCIÓN}

Identificar la percepción del nivel de empoderamiento de las enfermeras en el desarrollo de su quehacer, requiere de un ejercicio de análisis y reflexión a través de 
la aplicación de paquetes tecnológico- metodológicos pertinentes dentro de la investigación cualitativa, como son los grupos de discusión, entre otros, dado que éstos no siguen una metodología estadística o distributiva, sino estructural y abierta, pueden asimismo, reproducir exactamente al grupo social al que pertenecen, por ello sus disertaciones son atribuibles al gran discurso social a escala menor. Los grupos de discusión proporcionan datos cualitativos que a través de la reflexión y el diálogo aportan discurso oral sobre actitudes, percepciones, y opiniones de los agentes sociales a estudiar.

Caro y Vázquez (2002, p. 25), señalan que el término empoderamiento empezó a utilizarse a finales del siglo XVII, sin embargo en los últimos 15 años su uso parecía un arcaísmo lingüístico, no obstante, su pertinencia se ha generalizado en los diferentes escenarios y disciplinas configurándose como un concepto líder para integrar a los conceptos de participación, autonomía, identidad, desarrollo, integración y planeación; como también afirma Sánchez Herrera (2000) "que el empoderamiento conlleva una construcción colectiva, de autoimagen y confianza positivas, pensar críticamente construcción de cohesión de grupos, la toma de decisiones y la acción destacan como rasgos de este concepto", en este sentido, el empoderamiento conlleva la palabra poder que se relaciona con la motivación interna del y la profesional de enfermería así como con la política y lleva implícitos procesos educativos (Sánchez, 2000, p. 26).

La necesidad de un empoderamiento en la práctica de la enfermería, es uno de los retos que la han acompañado desde su origen, ya que esta profesión debe de posicionarse en todos los escenarios donde el cuidado se requiera. El abordaje de este término desde la disciplina y marco de referencia de enfermería a través de los grupos de discusión con quienes se trabajó en este estudio, propició la puesta en común de las diferentes interpretaciones y significados que de manera individual puedan prevalecer, ya que lleva implícitos elementos subjetivos, objetivos, políticos y sociales. Aunque el empoderamiento mediante procesos grupales puede ser altamente efectivo, finalmente puede llevar a cambios individuales reflejados en mayor autonomía, toma de decisiones y asertividad, por lo que resulta trascendental explorar la percepción y el nivel de empoderamiento que las enfermeras en servicio y los futuros profesionales de la enfermería hoy visualizan (Caro, 2006, p. 88).

Esta investigación forma parte de un proyecto Multipaís (México, Argentina y Chile), denominado "Comunicación, empoderamiento e identidad social de la enfermería en el contexto latinoamericano" en el que se plantea como pregunta central de investigación ¿Desde el punto de vista histórico y sociocultural, de qué manera la identidad social de la enfermería como profesión y la enfermera como sujeto social, están condicionadas estructuralmente en el nivel de empoderamiento de su ejercicio profesional, y en procesos de comunicación, información y género? (Bonilla, 2008, p. 5), en este artículo se presenta la sección correspondiente al empoderamiento de la profesión en Chihuahua, México.

Los supuestos hipotéticos que guiaron el análisis reflexivo fueron tres: uno, la relación enfermería como profesión y enfermera como sujeto social, dos, la existencia de conflicto de relaciones de poder y de comunicación al interior de las instituciones de 
salud, y tres, los conflictos de poder, provocan rupturas en el ejercicio de las funciones de la enfermera que están relacionados con la subordinación de las funciones de enfermería a las funciones de los médicos en instituciones hospitalarias. Los objetivos se centraron en identificar la percepción sobre la enfermería como profesión: (Estereotipos, motivaciones, representaciones, creencias, actitudes, valores y conductas manifiestas) así como reflexionar sobre la construcción social de la realidad por medio de un espacio de socialización como es el escenario de los Grupos de Discusión, en donde el discurso generado se deriva de la interacción de los participantes (Bonilla, 2008, p. 7).

\section{METODOLOGÍA}

Fue un estudio cualitativo, descriptivo y transversal, para el que se diseñó una estrategia metodológica triangulada con métodos cualitativos y cuantitativos, a través de la Entrevista en Profundidad, Encuesta y Grupos de Discusión, configurado en tres campos semánticos: Institución social, Identidad social y comunicación. Los resultados presentados en este artículo corresponden a la técnica de grupos de discusión de "empoderamiento del profesional de enfermería" ubicado en el campo semántico comunicación, el cual se exploró con una guía de discusión basada en detonadores previamente estructurados (Figura 3).

Se formaron dos grupos de discusión, conformados por enfermeras/os en activo, y dos de estudiantes de licenciatura en enfermería de primeros y últimos semestre, para el procesamiento y análisis de los datos se utilizó el protocolo de grupos de discusión, sin seguir una metodología estadística o distributiva, sino estructural y abierta, puede asimismo, reproducir exactamente al grupo social al que pertenecen, por ello sus disertaciones son atribuibles al discurso social en escala menor. Los grupos de discusión se conformaron con informantes clave, enfermeras-os que laboran en hospitales públicos y privados y por estudiantes de licenciatura en enfermería de los primeros y últimos semestres.

Los grupos de discusión proporcionaron información cualitativa sobre actitudes, percepciones, y opiniones de los agentes sociales que participaron, misma que en un primer momento se procesaron en una Matriz de Análisis Argumentativo (Bonilla, 2008), en un segundo momento, se identificaron objetos discursivos y tópicos a través de los cuales se reconstruyeron los argumentos del discurso de los informantes, y en un tercer momento, se construyó una gramática de argumentos o mapa ideológico (véase figura 1), en el cual se identificaron aspectos implícitos y explícitos del discurso de los participantes.

\subsection{Resultados}

Las enfermeras que laboran en el sector público y privado, llegaron al consenso de que la enfermera ejecuta el rol cuidador mediante la aplicación del proceso enfermero con bases teóricas sólidas, con lo que se posiciona profesional mente en el sector público y privado, aunque por otra parte identifican apatía en la toma de decisiones con mayor debilidad en los hospitales públicos, mientras que en los hospitales 
privados las decisiones que toman se encaminan al cuidado. "sabemos por qué lo estamos haciendo, vamos a brindar un buen servicio, un gran cuidado que esto va a repercutir en la salud, en la sociedad y en el profesional de enfermería..." (P. 1, 3, 2, $4,5)$.

Dos, en relación al rol como administrador, las enfermeras que están capacitadas en especialidades postécnicas son reconocidas para ocupar puestos administrativos de mandos medios. Tres, el rol docente la enfermera lo ejerce en los hospitales públicos, supervisando las prácticas estudiantiles, aunque "...lo hace sin deseos de participar en esta actividad..." como refirieron los participantes (P2,y4), afirmaron por igual, que la enfermera ejerce la función de educador a través de la orientación a los pacientes y sus familiares sobre cuidados de enfermería, también consideraron que el impacto de este rol se ubica en acciones preventivas comunitarias, que ejecuta en el primer nivel, "así tanto pienso que estamos ahí para educar a nuestros pacientes, familiares, siempre estamos educando..." ( $P 2,1,3,5,4)$, las enfermeras amplían sus posibilidades de consolidar el rol educativo y con ello contribuyen en la conservación de la salud de la población.

Cuatro, los participantes opinaron que, para ejercer el rol investigador es necesario tener competencias teórico-metodológicas, para aplicarlos en su campo profesional y que los resultados de este tipo de trabajos, aún no se ubican ampliamente en el ámbito de la enfermería, puesto que algunos estudios realizados por enfermeras apoyan el desarrollo de la medicina y han demostrado riesgos para el paciente en la práctica médica.

Cinco, en el ámbito institucional el empoderamiento político y administrativo, tanto en hospitales públicos como privados, se encontró que las políticas institucionales constituyen una limitante para empoderar a la enfermera, son una vía de empoderamiento para el gremio médico, quienes utilizan este poder para girar órdenes y por otra parte en el ámbito privado la presencia sindical no existe, sin embargo, la enfermeras afirman, a pesar de estos obstáculos cuentan con suficiente saber y motivación para realizar su trabajo e imprimirle valores como el respeto por la vida, la responsabilidad, identidad institucional y compromiso con la salud y las personas que cuidan, ver gramática de argumentos o mapa ideológico en la figura 1. 


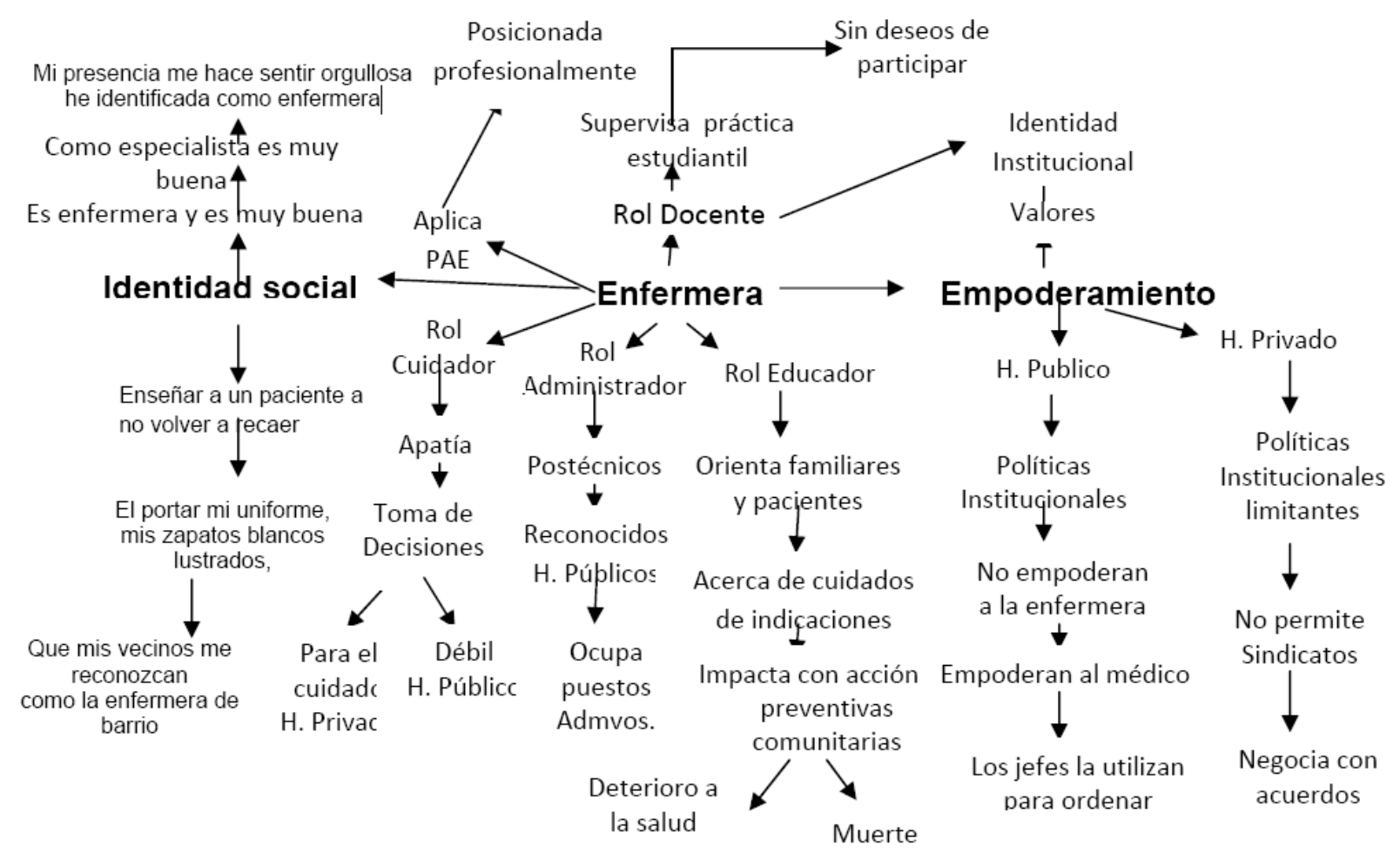

Figura 1: Gramática de Argumentos o Mapa ideológico sobre el discurso social general de enfermeras de hospitales públicos y privados de Chihuahua

Fuente: Grupo de Discusión Gral. de Enfermeras de Hospitales Públicos y privados. Chihuahua.

Estas expresiones coinciden con Blanchart, 1996) y Randolf (2001) (Citados por (Sánchez, 2002, p. 28), quienes exponen que "el empoderamiento no es darle poder a la enfermera, si ella cuenta con suficiente saber y motivación para hacer su trabajo de excelente forma", el empoderamiento es dejar que este poder salga a flote y se exprese. Una enfermera que está empoderada, tiene sentido de pertenencia, se siente propietaria de su práctica, se muestra comprometida con los usuarios de su servicio, sabe para dónde va. Resulta interesante en las innumerables acciones que realizan las enfermeras cotidianamente y ¿cuánto dolor mitigan? ¿Cuánto frío calman? ¿Cuánta soledad disminuye con su compañía? ¿Cuánto temor aminoran? ¿Cuánto momentos de cuidado establecen? y ¿cuantas acciones realizan a través del contacto y la sensibilidad humana propia de los profesionales de la enfermería?; sin embargo ante una omisión, la crítica es dura y se hace visible para opacar todo lo anterior.

\section{ANÁLISIS Y DISCUSIÓN}

Para el profesional de enfermería, la competencia está determinada por el conjunto de conocimientos, habilidades y actitudes, que definen la disciplina en el ámbito clínico y comunitario y se refleja en el empoderamiento profesional, las actitudes de responsabilidad y compromiso se articulan con las capacidades que se ponen en práctica para atender las necesidades sociales en el ámbito de cuidado que proporcionan a la persona, se constituye como el potencial humano más prometedor para la población y la propia institución. En este sentido las informantes del grupo de discusión coinciden en que existe "motivación, hacia buscar la profesionalización... relacionan teoría con práctica... búsqueda continua hacia mejorar el cuidado..." (p. 2, 
31,5 y 4). Esta forma de pensar y de actuar, resalta el desarrollo que tiene la profesión en las instituciones de salud.

La necesidad actual de adquirir conocimientos para mejorar el cuidado en la práctica de enfermería y expresada por los informantes no es aislada, "considero que nos preocupamos por tener más conocimientos y busco mejorar el cuidado con formación académica y práctica con bases teóricas"... (p. 3, 2, 4, 1,5), de ahí el interés personal y profesional para mejorar el cuidado a través de la profesionalización; las instituciones públicas favorecen este proceso, mediante cursos de mejora continua, becas y convenios con las Universidades, esto favorece el empoderamiento por conocimiento, base para la toma de decisiones dentro de su ámbito profesional, lo que concuerda con lo afirmado por (Cárdenas y Monroy, 2008, p. 39) Desde esta perspectiva la autonomía del ejercicio profesional de enfermería implica libertad para hacer juicios clínicos y tomar decisiones dentro del ámbito o alcance de la práctica profesional.

Hoy en día la dirección de las instituciones de salud continúan bajo la jerarquía del gremio médico, lo cual confiere poder a esta disciplina, asimismo el avance hacia el desarrollo profesional desde sus inicios como oficio, hasta ser reconocida, legalmente, y alcanzar el status de profesión por Decreto Presidencial publicado en el Diario Oficial de la Federación el 6 de Enero del 2005. Como profesión se consolida cuando se muestra: libertad de acción, toma de decisiones, responsabilidad de los resultados de su quehacer $\mathrm{y}$, autoridad en el proceso; en este sentido el grupo de discusión manifestó que: "las políticas favorecen a ser utilizadas para ejercer el poder", el personal del gremio médico y los jefes inmediatos aplican el liderazgo autocrático; en este sentido el reconocimiento de la profesión de enfermería en las instituciones de salud no ha trascendido, ya que "las políticas no empoderan a la enfermera, empoderan al médico y a los jefes". (P 4, 3,1, 5,2).

Por otra parte, las instituciones educativas formadoras del profesional de enfermería, para aprovechar la coyuntura del reconocimiento por decreto gubernamental y con el propósito de participar en el empoderamiento profesional e institucional, actualizan su Curriculum, como es el caso de la Facultad de Enfermería y Nutriología de la Universidad Autónoma de Chihuahua (UACh), que definió el perfil de egreso a la altura de las exigencias sociales e institucionales en el área de la salud y entre otras características menciona que prepara al licenciado en enfermería como un profesional de la salud con capacidad para ejercer los diferentes roles (Cuidador, Educador Investigador, Administrador y Colaborador), para proporcionar cuidado de enfermería a la persona, con base en la teoría de la Ciencia del Cuidado Humano de J. Watson (Watson, 1998) y para ejercer las competencias básicas, específicas y profesionales que le permitan el desempeño laboral con sentido ético y humanístico (Plan Curricular 2005).

Para avanzar hacia la consolidación de la profesión de enfermería, el grupo de discusión coincidió que la enfermera tiene bases sólidas de conocimientos y habilidades propias de la profesión; que la hacen trascender en beneficio de la persona al otorgar el cuidado; el cual tiene impacto en el sujeto de cuidado y la 
sociedad al contribuir en preservar la salud. "Sabemos por qué lo estamos haciendo, vamos a brindar un buen servicio un gran cuidado, que esto va a repercutir en la salud $y$, en la sociedad y en el profesional de enfermería" (P 1, 5, 3, 4, 2).

\section{CONCLUSIONES}

Las enfermeras/os no explicitan experiencias de empoderamiento, sin embargo a través de sus diálogos y discursos relatan e identifican vivencias que corresponden a atributos del empoderamiento, el futuro no invita a hacer enfermería sino a ser cada día mejores enfermeros(as) y a ser, como lo señala Alberdi 1993, citado por (Sánchez, 2002, p. 30), grandes en el arte, en la política, en la ciencia y en el negocio del cuidado. El empoderamiento ganado por las enfermeras está respaldado por el conocimiento, experiencia y compromiso profesional, el empoderamiento institucional se respalda en las políticas que favorecen el empoderamiento del gremio médico y obstaculizan el de las enfermeras.

\section{REFERENCIAS}

Bonilla, L. E. (2008). "Comunicación, empoderamiento e identidad social en el contexto latinoamericano". Proyecto de investigación multipaís, actualmente texto académico en proceso de publicación en coedición entre México, Chile y Argentina.

Cárdenas y Monroy, L. (2008). La enfermería mexicana en los albores del siglo XXI. Un análisis desde la sociología de las profesiones. Barcelona: Ediciones Pomares.

Caro, C. V. y Vázquez, M. L. (2000). El empoderamiento de y con enfermería. Cuidado y práctica de enfermería. Universidad Nacional de Colombia, Bogotá: UnibiblosEditorial Guadalupe.

Sánchez Herrera, B. (2002). Identidad y empoderamiento de la profesión de enfermería. Avances en Enfermería (Vol 20, No 1). Norteamérica, 20 sep. 2010. Consultado el 21 de Octubre del 2011, Disponible en:

www.revistas.unal.edu.co/index.php/avenferm/article/view/16310.

Watson, J. (1998). Enfermería: Ciencia Humana y Cuidado Humano: Una Teoría de Enfermería Naturaleza del Cuidado Humano y valores del cuidado en enfermería, en J. Watson (Ed.), Ciencia humana y cuidado humano. National League for Nursing

\section{B. Leticia Moriel Corral}

Docente de la Maestría en Enfermería de la Facultad de Enfermería y Nutriología de la Universidad Autónoma de Chihuahua. Integrante del Cuerpo Académico 102.

\section{Norma Pizarro}

Maestra en Enfermería. Docente de la Maestría en Enfermería de la Facultad de Enfermería y Nutriología de la Universidad Autónoma de Chihuahua. Integrante del Cuerpo Académico 102. Chihuahua. 


\section{Hilda Hernández Hernández}

Maestra en Enfermería. Coordinadora de Educación Continua Abierta y a Distancia en la Facultad de Enfermería y Nutriología de la Universidad Autónoma de Chihuahua. Líder del Cuerpo Académico 102. Chihuahua.

\section{Elizabeth Bonilla Loyo}

Dra. Administración y Ciencias Sociales por la Universidad La Salle, Cd. De México, DF. Maestra en Comunicación por la Universidad Iberoamericana, Cd. De México, DF. Licenciada en Psicología en la Universidad Veracruzana, Xalapa, Ver. Actualmente es investigadora de tiempo completo, Perfil Promep y Nivel IV de Productividad y Docente de la facultad de Enfermería de la Universidad Veracruzana. Responsable del proyecto de investigación "Comunicación, empoderamiento e identidad social de la enfermería en el contexto latinoamericano, y responsable del CA "Comunicación y educación en salud pública" del ISP de la Universidad Veracruzana, México. 